\title{
SPARSE IMAGE RECONSTRUCTION FOR PARTIALLY KNOWN BLUR FUNCTIONS
}

\author{
Raviv Raich and Alfred O. Hero III \\ Department of EECS, University of Michigan, Ann Arbor, MI 48109-2122, USA \\ \{ravivr, hero\}@ eecs.umich.edu
}

\begin{abstract}
In this paper, we consider the problem of image reconstruction from the noisy blurred version of an original image when the blurring operator is partially known and the original image is sparse. Using optimization transfer, we derive a novel iterative algorithm in closedform that incorporates both sparseness and partial knowledge of the image. We demonstrate the performance of the algorithm using simulations.
\end{abstract}

\section{INTRODUCTION}

Image deconvolution is a well-studied problem in image processing [1]. The task at hand is that of obtaining an image from a noisy blurred version. In the standard formulation of the problem, the blurring operator is a linear convolution of the original image with a known point spread function (PSF).

One modification of the original problem is considered when the PSF associated with the blurring operator is only partially known. Semi-blind reconstruction methods take advantage of partial knowledge of the PSF and try to account for the mismatch between the unknown true PSF to that of an approximated version of it based on partial knowledge.

An additional modification of the original problem we consider is when prior knowledge on the original image is available, specifically, when the image at hand is sparse. Most of the pixels of a sparse image are zero-valued and only a small number of its pixels are nonzero-valued. Sparseness of the image can occur not only in the image domain but also in some transform domain. Sparsity in the image domain occurs in molecular imaging and astronomy. In [2], penalized expectation maximization (EM) is used to separate the problem to iterative alternating steps of deconvolution and denoising problems. A sparse solution is obtained in the wavelet domain by adopting a sparsifying penalty. Additional references relating to sparse reconstruction are deferred to Section 2.2.

In this paper, we consider the deconvolution problem when the PSF of the blurring operator is partially known and the image is sparse in the image domain. These assumption can easily be extended to sparseness in other domains (e.g., wavelet, Fourier). We derive a novel iterative algorithm in closed-form as a solution to the problem and demonstrate the performance of the algorithm using simulations.

\section{PROBLEM FORMULATION}

We consider the problem of reconstructing a sparse blurred image in noise. First, we describe the non-sparse setup when some uncer-

This work was partially supported by the ARO MURI grant W911NF05-1-0403. tainty in the point PSF exists. Then, we extend the problem to the sparse setting.

\subsection{The Unconstrained Setup}

We start with the following model

$$
\mathbf{y}=\mathbf{H x}+\mathbf{n},
$$

where the original image is denoted by $\mathbf{x} \in \mathbb{R}^{n}$, the $m \times n$ blurring matrix is denoted by $\mathbf{H}$, the noise vector is denoted by $\mathbf{n} \in \mathbb{R}^{m}$, and the measured image is denoted by $\mathbf{y} \in \mathbb{R}^{m}$. Note that when the linear blurring operator $\mathbf{H x}$ describes a convolution, further structure is imposed on the matrix $\mathbf{H}$. When $\mathbf{n}$ is a zero-mean white Gaussian noise vector, i.e., $\mathbf{n} \sim \mathcal{N}\left(\mathbf{0}, \sigma^{2} \mathbf{I}\right)$, the maximum likelihood (ML) estimator of $\mathbf{x}$ is the minimizer of the following quadratic cost function

$$
J(\mathbf{x})=\|\mathbf{H} \mathbf{x}-\mathbf{y}\|^{2},
$$

which is also the least squares (LS) criterion. Note that we use $\|\cdot\|$ to denote the $l_{2}$ norm $\|\cdot\|_{2}$. Other norms will be written explicitly (e.g., $l_{1}:\|\cdot\|_{1}$ ).

The $l_{2}$-norm of a matrix $\mathbf{C}$ is given by $\|\mathbf{C}\|=\max _{\mathbf{s}}\|\mathbf{C s}\| /\|\mathbf{s}\|$. In this paper, we consider the case when the matrix $\mathbf{H}$ is only partially known and is given by $\mathbf{H}=\mathbf{H}_{0}+\epsilon \Delta$, where $\Delta$ has a unity $l_{2}$-norm:

$$
\|\Delta\| \leq 1
$$

and thus $\left\|\mathbf{H}-\mathbf{H}_{0}\right\| \leq \epsilon$. Therefore, the cost function in (2) can be written as

$$
J(\mathbf{x})=\left\|\left(\mathbf{H}_{0}+\epsilon \boldsymbol{\Delta}\right) \mathbf{x}-\mathbf{y}\right\|^{2},
$$

where $\Delta$ is unknown. To remove the dependence of the estimate of $\mathbf{x}$ on $\boldsymbol{\Delta}$, we consider the minimax criterion, i.e., we look for $\mathbf{x}$ that minimizes (4) for the worst case perturbation $\Delta$ so that $J(\mathbf{x})$ is now

$$
J(\mathbf{x})=\max _{\boldsymbol{\Delta}}\left\|\left(\mathbf{H}_{0}+\epsilon \boldsymbol{\Delta}\right) \mathbf{x}-\mathbf{y}\right\|^{2},
$$

subject to (3). The criterion in (5) can be simplified to $\left(\| \mathbf{H}_{0} \mathbf{x}-\right.$ $\mathbf{y}\|+\epsilon\| \mathbf{x} \|)^{2}$ [3, Ch. 6.4, p. 322], which is equivalent to

$$
J(\mathbf{x})=\left\|\mathbf{H}_{0} \mathbf{x}-\mathbf{y}\right\|+\epsilon\|\mathbf{x}\| .
$$

The criterion in (6) is a combination of the $l_{2}$-norm of two quantities. The first quantity is the $l_{2}$-norm of the error between the observations and their model based on the approximated forward operator $\mathbf{H}_{0}$. The second quantity is the $l_{2}$-norm of the solution and it offers regularization of the solution. Note that when $\epsilon>0$, the criterion in (6) uses the norm and not the squared norm and therefore is not quadratic. 


\subsection{The Sparse Setup}

Often a sparse solution is of interest. Such a solution has the property that only few of the elements of $\mathbf{x}$ are nonzero valued. Define the $l_{0}$ measure as

$$
\|\mathbf{x}\|_{0}=\sum_{i=1}^{m} I\left(\mathbf{x}_{i} \neq 0\right),
$$

where $I(\cdot)$ is the indicator function. With the $l_{0}$-constraint on $\mathbf{x}$, the problem of finding the optimal sparse image from the image blurred by a partially unknown blurring operator is given by

$$
\min _{\mathbf{x}}\left\|\mathbf{H}_{0} \mathbf{x}-\mathbf{y}\right\|+\epsilon\|\mathbf{x}\| \quad \text { s.t. }\|\mathbf{x}\|_{0} \leq p .
$$

This problem poses several difficulties. First, it is a combinatorial problem with possibly an exponential number of potential solutions. The number of possible solutions is $\left(\begin{array}{l}n \\ p\end{array}\right)$. Next, the problem in (8) is not convex, since $\left\{\mathbf{x}:\|\mathbf{x}\|_{0} \leq p\right\}$ is not a convex set. Therefore, solutions to (8) based on gradient or iterative methods are not guaranteed to converge to the global optimum.

Under certain conditions (on matrix $\mathbf{H}_{0}$ ), the non-convex $l_{0}$ constraint can be replaced by the convex $l_{1}$-constraint to yield the same solution. This concept is known as convex relaxation. The $l_{1}$-constrained formulation of the problem is given by

$$
\min _{\mathbf{x}}\left\|\mathbf{H}_{0} \mathbf{x}-\mathbf{y}\right\|+\epsilon\|\mathbf{x}\| \quad \text { s.t. } \quad\|\mathbf{x}\|_{1} \leq \gamma .
$$

When $\epsilon=0$, the problem reduces to a quadratic minimization subject to an $l_{1}$ constraint. This problem is a convex problem and is addressed in $[4,5]$. An iterative solution to the problem is presented in [6]. In [7], least angle regression is presented as a computationally efficient algorithm that provides the solution using the same complexity of the least squares solution, i.e., when no sparseness constraint is present.

When $\epsilon>0$, the criterion in (6) is no longer quadratic. However, it still preserves the convexity property. Convexity can be verified by obtaining the Hessian of the criterion in (6). The Hessian of the first term $\left\|\mathbf{H}_{0} \mathbf{x}-\mathbf{y}\right\|$ is given by

$$
\begin{aligned}
\frac{\partial^{2}\left\|\mathbf{H}_{0} \mathbf{x}-\mathbf{y}\right\|}{\partial \mathbf{x} \partial \mathbf{x}^{T}} & =\frac{\mathbf{H}_{0}^{T} \mathbf{H}_{0}}{\|\mathbf{e}\|}-\frac{\mathbf{H}_{0}^{T} \mathbf{e e}^{T} \mathbf{H}_{0}}{\|\mathbf{e}\|^{3}} \\
& =\frac{\mathbf{H}_{0}^{T}\left(\mathbf{I}-\frac{\mathbf{e} \mathbf{e}^{T}}{\|\mathbf{e}\|^{2}}\right) \mathbf{H}_{0}}{\|\mathbf{e}\|} \geq 0
\end{aligned}
$$

where $\mathbf{e}=\mathbf{H}_{0} \mathbf{x}-\mathbf{y}$. To verify that the matrix in (10) is positive semi-definite refer to [8]. Similarly, the Hessian of the second term $\|\mathbf{x}\|$ is positive semi-definite. This is trivial as $\|\mathbf{x}\|$ is a special case of $\|\mathbf{H x}-\mathbf{y}\|$ with $\mathbf{H}=\mathbf{I}$ and $\mathbf{y}=\mathbf{0}$.

\section{ALGORITHMS}

In the following, we recall the solution to the non-sparse problem and derive an iterative solution to the problem in (9), which incorporates a sparse constraint.

\subsection{Non-Sparse Solution - Tikhonov Regularization}

To minimize (6), we would like to find the optimal solution for each set $S_{E}=\left\{\mathbf{x}:\|\mathbf{x}\|^{2}=E, \mathbf{x} \in \mathbb{R}^{n}\right\}$ (i.e., for every $E$ ) independently, and then find $E$ that yields the overall optimal solution. For each set $S_{E}, \epsilon\|\mathbf{x}\|$ is constant and therefore minimization of (6) is equivalent to minimization of $\left\|\mathbf{H}_{0} \mathbf{x}-\mathbf{y}\right\|^{2}$ subject to $\|\mathbf{x}\|^{2}=E$, known as Tikhonov regularization [3, p. 306]. Using Lagrange multipliers, the problem becomes

$$
\hat{\mathbf{x}}=\arg \min _{\mathbf{x}}\left\|\mathbf{H}_{0} \mathbf{x}-\mathbf{y}\right\|^{2}+\lambda\|\mathbf{x}\|^{2},
$$

which has the following solution

$$
\hat{\mathbf{x}}=\left(\mathbf{H}_{0}^{T} \mathbf{H}_{0}+\lambda \mathbf{I}\right)^{-1} \mathbf{H}_{0}^{T} \mathbf{y} .
$$

Since we are looking for the optimal solution over all values of $E$, we therefore allow for all values of $\lambda \geq 0$ and thus the problem is reduced to the scalar minimization problem:

$$
\begin{array}{r}
\min _{\lambda}\left\|\left(\mathbf{H}_{0}\left(\mathbf{H}_{0}^{T} \mathbf{H}_{0}+\lambda \mathbf{I}\right)^{-1} \mathbf{H}_{0}^{T}-\mathbf{I}\right) \mathbf{y}\right\| \\
+\epsilon\left\|\left(\mathbf{H}_{0}^{T} \mathbf{H}_{0}+\lambda \mathbf{I}\right)^{-1} \mathbf{H}_{0}^{T} \mathbf{y}\right\| .
\end{array}
$$

The solution to (13) in terms of $\lambda$ is a function of $\epsilon, \mathbf{H}_{0}$, and $\mathbf{y}$. This problem involves finding the roots of a $4 \mathrm{~m}$-degree polynomial. Often, $\epsilon$ is not available and one can use alternative methods to find the parameter $\lambda$ (e.g., SURE based algorithm in [9]).

\subsection{Sparse Solution}

The solution we present in this section provides a generalization of the solution in Section 3.1 by incorporating sparseness. In [6], an iterative solution to (9) when $\epsilon=0$ is obtained using optimization transfer. Optimization transfer works in the following way. Denote the optimality criterion by $F(\mathbf{x})$. Let $Q\left(\mathbf{x}, \mathbf{x}^{\prime}\right)$ be a non-negative function, i.e., $Q\left(\mathbf{x}, \mathbf{x}^{\prime}\right) \geq 0$. Furthermore, $Q\left(\mathbf{x}, \mathbf{x}^{\prime}\right)=0$ for $\mathbf{x}=\mathbf{x}^{\prime}$. Consider the following iteration:

$$
\hat{\mathbf{x}}^{(n+1)}=\arg \min _{\mathbf{x}} F(\mathbf{x})+Q\left(\mathbf{x}, \hat{\mathbf{x}}^{(n)}\right) .
$$

For any $\mathbf{x}$, we have

$$
F\left(\hat{\mathbf{x}}^{(n+1)}\right)+Q\left(\hat{\mathbf{x}}^{(n+1)}, \hat{\mathbf{x}}^{(n)}\right) \leq F(\mathbf{x})+Q\left(\mathbf{x}, \hat{\mathbf{x}}^{(n)}\right) .
$$

Substituting $\mathbf{x}=\mathbf{x}^{(n)}$ in (15) and recognizing that $Q\left(\mathbf{x}, \hat{\mathbf{x}}^{(n)}\right)$ on the right hand side (RHS) is zero for $\mathbf{x}=\hat{\mathbf{x}}^{(n)}$, we obtain

$$
F\left(\mathbf{x}^{(n+1)}\right)+Q\left(\mathbf{x}^{(n+1)}, \mathbf{x}^{(n)}\right) \leq F\left(\mathbf{x}^{(n)}\right) .
$$

Since $Q\left(\mathbf{x}^{(n+1)}, \mathbf{x}^{(n)}\right) \geq 0$, we have

$$
F\left(\mathbf{x}^{(n+1)}\right) \leq F\left(\mathbf{x}^{(n)}\right) .
$$

This method guarantees that $F\left(\hat{\mathbf{x}}^{(n)}\right)$ is a non-increasing function of $n$. If $Q\left(\mathbf{x}, \mathbf{x}^{\prime}\right)$ satisfies $Q\left(\mathbf{x}, \mathbf{x}^{\prime}\right)>0$ for $\mathbf{x} \neq \mathbf{x}^{\prime}$, then $F\left(\hat{\mathbf{x}}^{(n)}\right)$ is a decreasing function of $n$.

Similar to the approach in Section 3.1, we consider solving for a strict $l_{2}$ constraint, i.e., $\|x\|^{2}=E$ and then optimizing the solution over $E$. The problem in (9) including the strict $l_{2}$-constraint can be phrased as

$$
\min _{\mathbf{x}}\left\|\mathbf{H}_{0} \mathbf{x}-\mathbf{y}\right\|, \quad \text { s.t. } \quad\|\mathbf{x}\|^{2}=E \text { and }\|\mathbf{x}\|_{1}=L,
$$

which is equivalent to

$$
\min _{\mathbf{x}} F(\mathbf{x}) \quad F(\mathbf{x})=\left\|\mathbf{H}_{0} \mathbf{x}-\mathbf{y}\right\|^{2}+\lambda_{1}\|\mathbf{x}\|^{2}+\lambda_{2}\|\mathbf{x}\|_{1},
$$

subject to $\|\mathbf{x}\|^{2}=E$ and $\|\mathbf{x}\|_{1}=L$. Denote the largest singular value of $\mathbf{H}_{0}$ by $s$. Let

$$
\begin{aligned}
Q\left(\mathbf{x}, \mathbf{x}^{\prime}\right) & =s^{2}\left\|\mathbf{x}-\mathbf{x}^{\prime}\right\|^{2}-\left\|\mathbf{H}_{0}\left(\mathbf{x}-\mathbf{x}^{\prime}\right)\right\|^{2} \\
& =\left(\mathbf{x}-\mathbf{x}^{\prime}\right)^{T}\left(s^{2} \mathbf{I}-\mathbf{H}_{0}^{T} \mathbf{H}_{0}\right)\left(\mathbf{x}-\mathbf{x}^{\prime}\right) .
\end{aligned}
$$


Since $s$ is the largest singular value of $\mathbf{H}_{0},\left(s^{2} \mathbf{I}-\mathbf{H}_{0}^{T} \mathbf{H}_{0}\right)$ is a positive semi-definite matrix and thus $Q\left(\mathbf{x}, \mathbf{x}^{\prime}\right)$ satisfies: $Q\left(\mathbf{x}, \mathbf{x}^{\prime}\right) \geq 0$ and $Q\left(\mathbf{x}, \mathbf{x}^{\prime}\right)=0$ for $\mathbf{x}=\mathbf{x}^{\prime}$. Since the criterion in (9) is convex, convergence to the global solution is achievable. Substituting $F(\mathbf{x})$ from (18) and $Q(x)$ from (19) into (14) and simplifying, we obtain

$$
\hat{\mathbf{x}}^{(n+1)}=\arg \min _{\mathbf{x}}\left(\lambda_{1}+s^{2}\right) \mathbf{x}^{T} \mathbf{x}-2 \mathbf{x}^{T} \mathbf{v}^{(n)}+\lambda_{2}\|\mathbf{x}\|_{1},
$$

where $\mathbf{v}^{(n)}=s^{2} \hat{\mathbf{x}}^{(n)}+\mathbf{H}_{0}^{T}\left(\mathbf{y}-\mathbf{H}_{0} \hat{\mathbf{x}}^{(n)}\right)$. The minimization in (20) can be implemented by minimizing w.r.t each $\mathbf{x}_{i}$ independently

$$
\hat{\mathbf{x}}_{i}^{(n+1)}=\arg \min _{\mathbf{x}_{i}}\left(\lambda_{1}+s^{2}\right) \mathbf{x}_{i}^{2}-2 \mathbf{x}_{i} \mathbf{v}_{i}^{(n)}+\lambda_{2}\left|\mathbf{x}_{i}\right| .
$$

The solution to (21) is given by

$$
\hat{\mathbf{x}}_{i}^{(n+1)}=f_{\lambda_{2} /\left(s^{2}+\lambda_{1}\right)}\left(\mathbf{v}_{i}^{(n)} /\left(s^{2}+\lambda_{1}\right)\right),
$$

where

$$
f_{\delta}(\xi)=\left(\xi-\operatorname{sgn}(\xi) \frac{\delta}{2}\right) I\left(|\xi|>\frac{\delta}{2}\right)
$$

is known as the soft-threshold function. Let $\tilde{\mathbf{H}}_{0}=\mathbf{H}_{0} / s$, the solution can be written compactly as

$$
\hat{\mathbf{x}}_{i}^{(n+1)}=\alpha f_{\delta}\left(\left[\mathbf{x}^{(n)}+\tilde{\mathbf{H}}_{0}^{T}\left(\mathbf{y} / s-\tilde{\mathbf{H}}_{0} \mathbf{x}^{(n)}\right)\right]_{i}\right),
$$

where $\alpha=\frac{s^{2}}{s^{2}+\lambda_{1}} \leq 1$ and $\delta=\frac{\lambda_{2}}{s^{2}+\lambda_{1}}$. We would like to point out that in [10] an iterative algorithm similar to (24) was derived based on an $l_{p}$ constraint $(p<1)$.

Finally, one can find the optimal values for $\delta$ and $\alpha$ by evaluating our criterion over $\alpha$ and $\delta$. If $\epsilon$ is unknown and $\gamma$ in (9) is arbitrarily chosen, obtaining the optimal values for $\alpha$ and $\delta$ is not obvious. As in Section 3.1, other criteria can be used to determine $\alpha$ and $\delta$ based on the data (e.g., SURE based algorithm in [9]).

\section{SIMULATIONS}

In this section, we investigate the performance of the algorithm presented in Section 2 by simulation using Matlab ${ }^{\circledR}$. The algorithm in (24) is given in matrix/vector format. While in our derivations we use vectors to represent both the original image and the noisy blurred image, the algorithm we derived can be easily applied to two-dimensional images by representing the images as vectors. The operator $\mathbf{H x}$ corresponds to a two-dimensional convolution of the PSF associated with $\mathbf{H}$ and the image associated with $\mathbf{x}$. Similarly, the operator $\mathbf{H}^{T} \mathbf{y}$ corresponds to a two-dimensional convolution of the PSF associated with $\mathbf{H}$ flipped along both axes with the image associated with $\mathbf{y}$.

We generate the original image (represented by $\mathbf{x}$ ) as a $32 \times 32$ image with all but 10 zero valued pixels (see Fig. 3). By a random permutation, we select the positions of the 10 nonzero values and their values are all 1 . The PSF corresponding to the matrix $\mathbf{H}$ and the PSF corresponding to its approximation $\mathbf{H}_{0}$ are presented in Fig. 1(a) and Fig. 1(b), respectively. The circular PSF we adopt for the simulation (Fig. 1(a)) is an idealized two-dimensional magnetic resonance force microscopy (MRFM) PSF (one in which the cantilever is an ideal cylindrical spindle with symmetric stiffness coefficient). This differs from the PSF in [11] only in that the cantilever is modeled as a flattened spindle that has higher stiffness for motion along perpendicular to the flat edge. The resulting image $\mathbf{y}$ (see Fig. 4) is generated by convolving the original image $\mathbf{x}$ with the PSF corresponding to $\mathbf{H}$ and adding zero mean white Gaussian noise with standard deviation of $\sigma=0.2$.

First, we investigate our algorithm by computing the sum of squared error (SSE) $\|\hat{\mathbf{x}}-\mathbf{x}\|^{2}$ as a function of the parameters of the algorithm $\alpha, \delta$. To obtain $\hat{\mathbf{x}}$, we run our algorithm with 10,000 iterations. We fix $\delta$ at 0.005 , and vary $\alpha$. Figure 2 depicts the SSE vs. $1-\alpha$ on a logarithmic scale. We observe that the SSE reaches a minimum around $\alpha=0.99$. At this value the SSE is approximately 9.5. When $\alpha \rightarrow 1$, the SSE is approximately 15.5 . The value $\alpha=1$ corresponds to $\lambda_{1}=0$ or $\epsilon=0$, which is the case where the algorithm is not trying to correct for the mis-modeling error. The difference in the resulting SSE is obviously significant. Thus appropriately accounting for the mis-modeling error yields an improved performance in terms of SSE.

Next, using the setting in the previous paragraph, we would like to visually compare the resulting reconstructions at $\alpha=1$ and at $\alpha=0.99$. Figure 5 presents the sparse reconstruction by (24) with $\alpha=1$ and $\delta=0.005$. Figure 6 presents the sparse reconstruction by (24) with $\alpha=0.99$ and $\delta=0.005$. We make the following observations:

- Figure 5 presents pixels with larger values in the location of the original nonzero coefficients. Figure 6 presents pixels with smaller values in the location of the original nonzero coefficients. Since the $l_{2}$-norm constraint $\epsilon\|\mathbf{x}\|$ in (9) is present only in the reconstruction yielding Fig. 6 , pixels values in general are smaller than those in Fig. 5 since the $l_{2}$ constraint tends to reduce the magnitude of the solution.

- The number of nonzero coefficients in Fig. 5 is smaller than that in Fig. 6 . The additional $l_{2}$-norm constraint present in the reconstruction in Fig. 6 yields in general results that are less sparse.

We make the conclusion that the algorithm in (24) with $\alpha<1$ yields a more conservative reconstruction as compared with the case of $\alpha=$ 1.

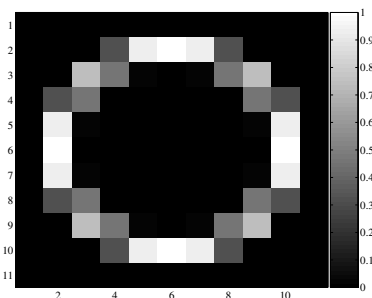

(a) The true PSF $\mathbf{H}$.

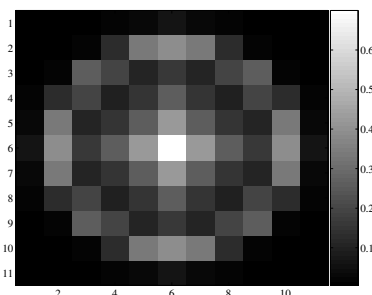

(b) The approximate PSF $\mathbf{H}_{0}$.
Fig. 1. The true and approximate PSFs

\section{CONCLUSIONS AND FUTURE WORK}

We presented a formulation of the deconvolution problem when the original image is sparse and the PSF of the blurring operator is only partially known. We derived a novel iterative solution to the problem based on optimization transfer. Using simulations, we illustrated the behavior of the algorithm in two cases: when mis-modeling is assumed and when no mis-modeling is assumed. When mis-modeling is present, the algorithm that assumes mis-modeling achieves a smaller SSE. Future work remaining to be addressed is the determination of the parameters of the algorithm (i.e., $\alpha$ and $\delta$ ). 


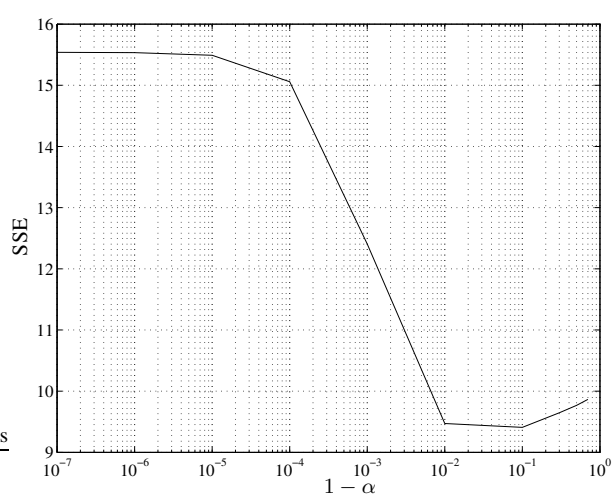

Fig. 2. The SSE of the image reconstructed using (24) with $\delta=$ 0.005 vs. $\alpha$.

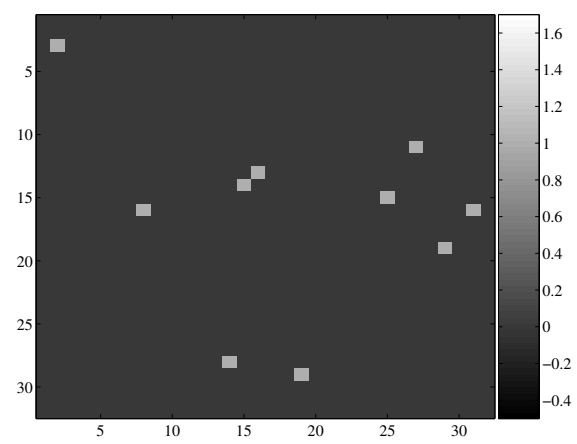

Fig. 3. The original sparse image. This image has 10 nonzero-valued pixels and their positions are selected by a random permutation.

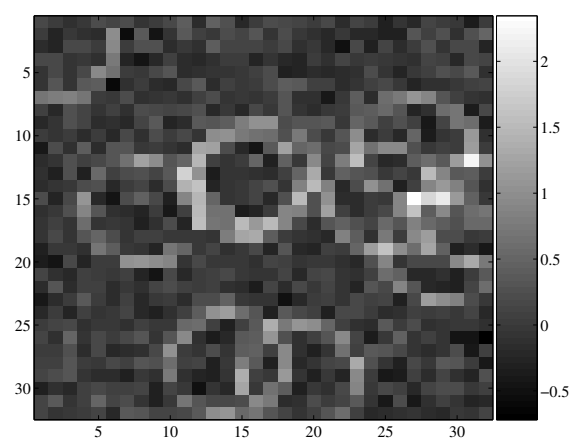

Fig. 4. The noisy blurred image. This image generated by convolving the image in Fig. 3 with the PSF in Fig. 1(a) and adding zero mean white Gaussian noise with standard deviation of 0.2 .

\section{REFERENCES}

[1] H. Andrews and B. Hunt, Digital Image Restoration. Englewood Cliffs, NJ: Prentice-Hall, 1977.

[2] M. A. T. Figueiredo and R. D. Nowak, "An EM algorithm for wavelet-based image restoration," IEEE Transactions on Image Processing, vol. 12, pp. 906-916, Aug. 2003.

[3] S. Boyd and L. Vandenberghe, Convex Optimization. New

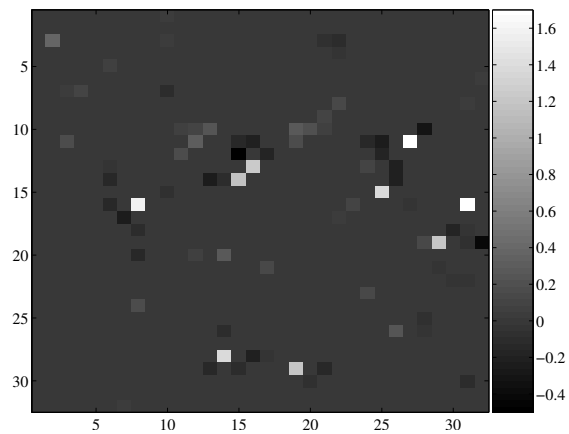

Fig. 5. The reconstructed image using (24) with $\alpha=1, \delta=0.005$ and the PSF in Fig. 1(b). SSE $=15.5$.

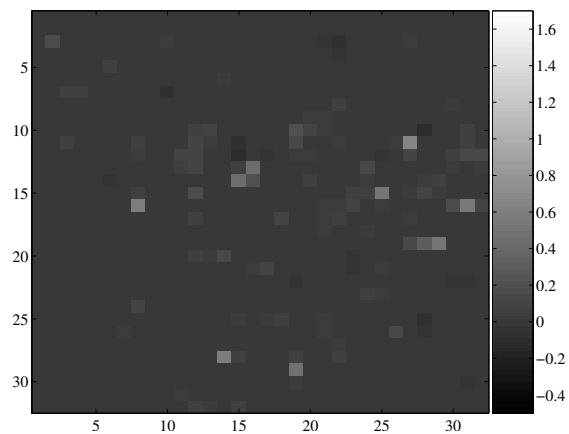

Fig. 6. The reconstructed image using (24) with $\alpha=0.99, \delta=$ 0.005, and the PSF in Fig. 1(b). SSE $=9.5$.

York, NY: Cambridge University Press, 2004.

[4] S. Alliney and S. A. Ruzinsky, "An algorithm for the minimization of mixed $l_{1}$ and $l_{2}$ norms with application to Bayesian estimation,” IEEE Trans. Signal Processing, vol. 42, pp. 618-627, Mar. 1994.

[5] R. Tibshirani, "Regression shrinkage and selection via the lasso," J. Royal. Statist. Soc B., vol. 58, no. 1, pp. 267-288, 1996.

[6] I. Daubechies, M. Defrise, and C. D. Mol, "An iterative thresholding algorithm for linear inverse problems with a sparsity constraint," Comm. Pure App. Math., vol. 57, pp. 1413-1457, Nov. 2004.

[7] B. Efron, T. Hastie, I. Johnstone, and R. Tibshirani, "Least angle regression,” Ann. Statist., vol. 32, no. 2, pp. 407-499, 2004.

[8] G. Strang, Linear Algebra and Its Applications. Philadelphia, PA: Saunders, third ed., 1988.

[9] V. Solo, "A SURE-fired way to choose smoothing parameters in ill-conditioned inverse problems," in Proc. IEEE Int. Conf. Image Processing, vol. 3, pp. 89-92, Sept. 1996.

[10] M. A. T. Figueiredo and R. D. Nowak, "A bound optimization approach to wavelet-based image deconvolution," in Proc. IEEE Int. Conf. Image Processing, vol. 2, pp. 782-785, Sept. 2005.

[11] M. Ting, R. Raich, and A. O. Hero, "A Bayesian approach to sparse image reconstruction.” Draft of paper in preparation. 